\title{
Research on the Electrocardiogram Manifestations of Acute Urticaria
}

\author{
Feng Chen ${ }^{1}$, Zhen Tian ${ }^{2}$ \\ ${ }^{1}$ Dermatology Department, China-Japan Union Hospital of Jilin University, Changchun City, Jilin Province, \\ 130033, China \\ ${ }^{2}$ Cardiology Department, China-Japan Union Hospital of Jilin University, Changchun City, Jilin Province, \\ 130033, China
}

Keywords: Electrocardiogram; Manifestations; Acute Urticaria

\begin{abstract}
Urticaria, commonly known as rubella mass, is a transient localized edema response caused by small vasodilation and increased permeability of the skin and mucosa, and it is a common allergic disease of skin and mucosa. Its etiology is complex, including food, medicine, infection, physicochemical factors, animal and plant factors, mental factors, systemic diseases, genetic factors. The most important clinical manifestation of urticaria is wind-mass and most patients feel severe itching, and wind-mass usually last for several hours, but it is prone to recurrent attacks. Urticaria can be divided into acute urticaria and chronic urticaria. The course of disease was acute urticaria at less than 6 weeks and chronic urticaria at or over 6 weeks. Acute urticaria is a common disease in dermatological clinic. From October 2015 to May 2018, 67 patients with acute measles were examined by electrocardiogram before and after treatment to investigate the relationship between ECG changes and other clinical symptoms in patients with Urticaria.
\end{abstract}

\section{Clinical Data}

\subsection{General information.}

A total of 67 patients with acute urticaria were diagnosed clinically, including 37 males and 30 females, aged between 12 and 68 years, with an average age of (32 \pm 2$)$ years. There were 24 cases with palpitation, 24 cases with discomfort in the anterior region, 10 cases with abdominal pain, 6 cases with infectious urticaria and 3 cases with drug urticaria. There were 5 cases with coronary heart disease (CHD) or myocardial ischemic electrocardiogram, but 67 cases had no typical angina pectoris before and after the onset.

\subsection{Method.}

67 patients had skin itching on admission and repeated 12-lead electrocardiogram examination after the wind mass disappeared. All patients were given oral administration of two antiallergic drugs. In the case of drug-induced urticaria, the sensitizers should be stopped first. In addition to antiallergic drugs, hormone therapy (dexamethasone) should be given and then reduced to stop as appropriate. Infectious urticaria is administered orally or intravenously with antibiotics. In addition, there is a need for appropriate symptomatic treatment, such as suffocation given oxygen; if abdominal pain is significant, we give the patient antispasmodic.

\section{Results}

In the 67 patients, 23 had electrocardiogram changes at the time of onset, and the ECG returned to normal after recovery. Among them, 19 cases had tachycardia, 1 case had arrhythmia, 1 case had frequent ventricular premature, 1 case had right deviation of electric axis, and 1 case had abnormal $\mathrm{P}$ wave. The electrocardiogram was abnormal in 9 patients with urticaria, including 2 tachycardia, 1 bradycardia, 1 atrial premature beat, 3 limb low voltage, 2 left ventricular high voltage, 2 left axis deviation. One case was translocated in the clockwise direction, and one case was in P-R interval. 35 cases had sinus rhythm and normal electrocardiogram at the time of onset and after treatment. 


\section{Discussion}

At present, western medicine for urticaria is mostly antihistamine, calcium, vitamin, immunosuppressant, etc., which has the disadvantages of poor long-term effect and high recurrence rate. Traditional Chinese medicine (TCM) has been treating urticaria for thousands of years, so this treatment has been paid more and more attention because of its rich clinical experience, small side effect and remarkable long-term effect. The incidence of urticaria is increasing year by year, which seriously affects the quality of life of patients. Therefore, it is an inevitable trend of clinical and basic research to explore a reliable and effective prescription for the treatment of urticaria. Urticaria is a localized edema response due to skin and mucosal vasodilation and increased permeability. Acute urticaria is a common clinical disease characterized by itching wind-mass or allergic dermatosis with vascular edema. It is a localized edema reaction due to the dilatation and permeability of small blood vessels of skin and mucous membrane. Urticaria usually subsided at 24 hours, however, in the case of recurrent rashes, the course of the disease is delayed by days to months. The main causes of acute urticaria are food and food additives, inhalation, infection, drugs, physical factors such as mechanical stimulation, heat and cold, sunlight, insect bites, mental factors, endocrine changes and genetic factors. Typical clinical symptoms include itchy skin, tubule, papules, etc. The tubules can be fused into large lesions, or map-like lesions, showing red bloated patches.For wind-like dermatitis, multiple itchy erythematodermatitis and perineum dermatitis, drugs such as diphenhydramine hydrochloride, chlorphenamine and prednisone acetate can be used to relieve itching. With the increase of dermatitis, the amount of medicine should be increased properly. Meanwhile, the patients should be told to bathe and change their trousers, and to scratch less, so as to prevent skin damage and chronic eczema.

Cardiac urticaria, also called myocardial vascular neuroedema, can be caused by allergic reactions such as food, medicine, pollen and so on. It often occurs at the same time with skin urticaria, but it also has individual differences. It was suggested that heart disease in urticaria could occur in the following ways: It causes coronary artery spasm, coronary artery edema, myocardial urticaria, myocardial vascular neuroedema, which leads to insufficiency of coronary artery blood supply, decrease of myocardial compliance, and decrease of blood pressure due to weakened contractility. Clinical symptoms are chest tightness, palpitation, chest pain, angina pectoris, arrhythmia, heart failure. Electrocardiogram can show different degree of ST-T changes and various arrhythmias.

Some scholars think that the change of ST-T can be regarded as the main reliable index of cardiac urticaria. When the coronary artery edema is more acute, it can cause acute myocardial infarction. The extensive ischemic electrocardiogram can see the pattern of acute myocardial infarction and it is easy to be misdiagnosed and mistreated. In previous reports, abnormal electrocardiogram and myocardial enzymes and myocardial protein were found in normal range. If electrocardiograms were found, myocardial enzymes and myocardial proteins were abnormal, it might be related to the involvement of various mechanisms of immune injury and increased vascular permeability.

Due to the development and application of new drugs, allergic reactions caused by drugs have increased, especially in allergic physique. Therefore, in clinical nursing care, it is necessary to closely observe the various reactions of the patients during the course of medication. Once urticaria occurs at the same time as chest tightness, palpitations, shortness of breath or even chest pain, and other heart diseases are excluded, the drug should be stopped immediately and the doctor should be informed, and an electrocardiogram needs to be done immediately. Once the diagnosis is established, it is necessary to use adrenaline, antihistamine drugs and glucocorticoids, and antishock therapy should be given when necessary to control ischemic heart damage caused by urticaria. For the early appearance of acute inferior wall myocardial infarction pattern, after active anti-allergic anti-shock treatment, cardiac symptoms and signs were effectively improved in a short time, and changes of electrocardiogram and myocardial enzyme ischemia returned to normal within a few days. Therefore, cardiac urticaria has the characteristics of temporary and reversible changes in myocardial ischemic injury and electrocardiogram. It is of certain significance to correctly 
recognize cardiac urticaria and observe the dynamic changes of ECG in clinical nursing.

In recent years, more and more clinical attention has been paid to cardiac damage caused by acute urticaria. Most of acute urticaria were type I allergy. The main pathogenesis is that a few allergic constitution cholinergic excitability is increased due to the lack of certain enzymes or secretory IgA in the body, and the body loses the ability to decompose and inactivate the chemical medium released by the allergic reaction. In addition to damaging the skin and mucous membrane, these mediators act directly on the cardiovascular system, causing myocardial capillaries to dilate, increase permeability, plasma exosmosis, myocardial interstitial inflammatory edema, and thus it will affect the repolarization process of the myocardium and the heart conduction system, so a series of ECG changes appeared.

Electrocardiogram changes caused by acute urticaria are easily confused with acute myocarditis, coronary heart disease, angina pectoris and so on. According to the clinical manifestation and related laboratory examination, it is not difficult to distinguish with it. In the basic unit, due to lack of understanding, palpitations, chest tightness and other mistaken throat mucosal, bronchial mucosal edema, so it often ignores electrocardiogram examination. Therefore, for patients with acute urticaria such as palpitation, chest tightness, dyspnea and other symptoms and signs, they should be routine electrocardiogram: if there are changes in electrocardiogram, in addition to timely antiallergic treatment, it is necessary to apply the drug to relieve cardiac load.

Electrocardiogram of 67 patients with acute urticaria was observed and analyzed in our hospital. Of the 67 patients, 23 had electrocardiogram changes at the time of onset, and the ECG returned to be normal after recovery. Among them, 19 cases of tachycardia, 1 case of arrhythmia, 1 case of frequent ventricular premature, 1 case of right deviation of electric axis, 1 case of abnormal $P$ wave. The electrocardiogram was abnormal in 9 patients with urticaria, including 2 tachycardia, 1 bradycardia, 1 atrial premature beat, 3 limb low voltage, 2 left ventricular high voltage, 2 left axis deviation. 1 case in the clockwise direction, 1 case in the P-R interval, and 1 case in the maximum value of the P-R interval.

The electrocardiogram was abnormal in 9 patients with urticaria, including 2 tachycardia, 1 bradycardia, 1 atrial premature beat, 3 limb low voltage, 2 left ventricular high voltage, 2 left axis deviation, 1 case in the clockwise direction, 1 case in the P-R interval, and 1 case in the maximum value of the P-R interval. 35 cases had sinus rhythm and normal electrocardiogram at the time of onset and after treatment.

Cardiac damage in urticaria is caused by cardiac capillary dilatation, increased permeability and edema in the interstitial of the heart. It may show symptoms such as palpitation, chest tightness, chest pain or discomfort in the precardiac area. The electrocardiogram may show a variety of abnormalities. Therefore, it is necessary to examine ECG regularly in acute urticaria. The causes of abnormal ECG changes in patients with urticaria are mainly related to the following factors: (1) Histamine levels in the body are elevated during the onset of urticaria, which can also lead to coronary vasospasm of the heart. Because of systemic capillary dilatation and increased vascular permeability, the effective circulation blood volume is reduced and myocardial blood supply is insufficient, which results in abnormal changes of electrocardiogram. (2) In some patients with special type of urticaria, in addition to histamine level, there were also changes of vasoactive substances such as acetylcholine or epinephrine, which could cause myocardial insufficiency. (3) Some patients had a history of cardiovascular diseases before onset, and even the changes of light vasoactive substances were more sensitive to the occurrence of urticaria, which could induce or aggravate myocardial insufficiency and lead to abnormal changes of electrocardiogram.

In a word, because many factors can cause abnormal electrocardiogram changes in patients with urticaria, we should attach great importance to it in clinic, especially for some old patients who have a history of coronary heart disease in case of cardiovascular accident.

\section{References}

[1] Zou Jinbo, Lu Tao, Zhang Shuping. Clinical analysis of 82 cases of acute urticaria[J]. Chinese Practical Medicine, 2015, 10 (19): 198-1999. 
[2] Sun Zhenyan, Zhang Jing, Zheng Jianfeng.Clinical analysis of 133 cases of acute urticaria[J]. Journal of contemporary clinical medicine, 2015, 28(03): 1374-1375.

[3] Lian Xiaoli, Zhou Zhike. A retrospective analysis of myocardial enzyme spectrum in 45 patients with acute urticaria [J]. Journal of diagnosis and treatment of Dermatology and venereal Diseases, 201825 (02): 78-80.

[4] Xu Zhiyou, Sun Qiwei. Clinical manifestation of 253 cases of acute urticaria and analysis of related laboratory examination [J]. Chinese community physician, 2014, 30(28): 129-130.

[5] Peng Li, Han Zhaoxia, Peng Xiaoming. Analysis of blood routine and C reactive protein in 549 patients with acute urticaria[J]. Chinese Journal of Dermatology and Venereology, 2017, 31 (10): 1091-1093. 